ORIGINAL ARTICLE

\title{
Moderate movement variability is optimal in massive practiced dart throws
}

\author{
Mahdi Nabavinik ${ }^{1 \mathrm{ABDE}}$, Hamideh Abdolzadeh ${ }^{2 \mathrm{CDE}}$ \\ ${ }^{1}$ University of Mazandaran, Iran \\ ${ }^{2}$ University of Vali-Asr Rafsanjan, Iran
}

Authors' Contribution: A - Study design; B - Data collection; C - Statistical analysis\#; D - Manuscript Preparation; E - Funds Collection.

\begin{tabular}{|c|c|}
\hline \multicolumn{2}{|l|}{ Abstract } \\
\hline Purpose: & $\begin{array}{l}\text { Movement variability is one of the fundamental topics concerning the control of human movement. } \\
\text { In recent years, researches have focused on various aspects of variability, which has changed the noise } \\
\text { to useful variable on human movement. Present study investigated movement variability level in high } \\
\text { skilled dart players that repeated throws over many years. }\end{array}$ \\
\hline Material: & $\begin{array}{l}\text { Seven experienced dart players (three women and four men) were threw } 36 \text { darts in three sets (each set } \\
12 \text { throws) from a standard distance ( } 2.37 \text { meters), while the kinematic features of the shoulder, elbow, } \\
\text { and wrist were recorded during the throws. Qualisys motion capture system with six cameras was used } \\
\text { to record the kinematics of the elbow. }\end{array}$ \\
\hline Results: & $\begin{array}{l}\text { Entropy analysis revealed that greater variability in movement angle, velocity and acceleration resulted } \\
\text { in better dart throwing performance but after reach to this level, throw variability was decreased. The } \\
\text { remarkable point in these findings was that variability was constant across all samples despite the varied } \\
\text { range of experience in throwing darts from } 2.37 \text { meters distance. Entropy analysis showed that in the } \\
\text { throws of highly experienced individuals, variation led to greater throwing efficiency. }\end{array}$ \\
\hline Conclusions: & $\begin{array}{l}\text { These findings suggest that variability in a throwing activity, which revealed that moderate movement } \\
\text { variability results in optimal throwing performance when dart throw has massive amount of practice } \\
\text { during many years. }\end{array}$ \\
\hline Keywords: & motor variability, dart throw, optimal performance, moderate variability, kinematics. \\
\hline
\end{tabular}

\section{Glossary}

Movement variability: Movement variability normal variations that occur in motor performance across multiple repetitions of a task.

Kinematic: Kinematics is a subfield of classical mechanics that describes the motion of points, bodies (objects), and systems of bodies (groups of objects) without considering the forces that cause them to move.

Generalized motor program: A generalized motor program is thought to develop over practice and provides the basis for generating movement sequences within a class of movements that share the same invariant features, such as sequence order, relative timing, and relative force. Dynamical system theory: The basic premise is that movement behavior is the result of complex interactions between many different subsystems in the body, the task at hand, and the environment.

Kinematic variability: Kinematic variability was computed as the average SD (Mean SD) of acceleration patterns among some movement repetitions.

Uncontrolled manifold: A hypothesis proposes that the central nervous system does not eliminate the redundant degrees of freedom, but instead it uses all of them to ensure flexible and stable performance of motor tasks.

Degrees of freedom: Degree of freedom of a system is the number of parameters of the system that may vary independently.

Throwing accuracy: It refers to that throwing score that

(c) Mahdi Nabavinik, Hamideh Abdolzadeh, 2020

doi:10.15561/26649837.2020.0604 more near to triple 20

Joint kinematic: Joint kinematics is the relative motion between two consecutive segments of the human that here is elbow joint.

Entropy: Entropy analysis is applicable to a larger class of problems than is usually studied through the use of mean square error analysis and yields the classical results when applied to Gaussian-linear systems.

Sagittal plane: The sagittal plane is an anatomical boundary that exists between the left and right sides of the body.

Trajectory: Trajectory refers to the marker elbow path during dart throw.

Round: Every three dart throws means one round in dart rules.

Triangle 20: There is one triangle of 20 score in which one dart has multiply in 3 .

\section{Introduction}

Movement variability is considered as a regular change in human movement and can be defined as normal changes during repetitions of a motion [1]. If a darts player completes five dart throws from the same distance, none of the throws would be identical to one another as changes occur at different levels of the nervous system. In this regard, movement variability has been investigated and reviewed based on three general perspectives. Firstly, from the perspective of the generalized motor program (GMP), more changes in new movements, the 
greater the movement learning, and movement schemata learning will be [2]. In other words, increased movement variability is considered advantageous, and the stability of repetitions is a relatively negative factor in learning. The uncontrolled manifold (UCM) hypothesis comprises the second major viewpoint regarding movement variability. The basis of this hypothesis is the unlimited degrees of freedom and movement solutions in the nervous system, stating that the movement system always has excessive components and solutions that can easily solve any momentary problem in movement [3]. The third view on movement variability is the dynamic systems theory, which holds that the movement system is in a steady state prior to learning a new movement. When a new variable is added to the system, the system enters chaos, before arriving at another period of stability; this process is called self-organization and forms the basis of many changes in the human nervous system [4]. The commonality between all three theories on human movement variability is that whenever variability decreases, performance enhances and the system quality increases [5]. Nevertheless, some arguments have been made in the literature about whether movement variability is good or bad [6]. Findings from clinical trials indicate that movement disorders in certain diseases can increase the variability of movements. In this case, increased variability is indicative of decreased performance quality [7]

However, research findings have not always been against movement variability. Some researchers have reported that increased variability at various levels of the motor system helps to increase performance quality. Zoffoli et al [8] investigated the kinematic variability of walking, and, by placing accelerometers on $\mathrm{C} 7$ and S2, concluded that walking with the aid of a pole can increase walking quality and variability at different speeds. In contrast, Qiao et al. [9] investigated the kinematic variability of the gait of aged subjects, and concluded that kinematic variability depends on the phases of gait, with the greatest variability occurring during the pushup and swing phases. Aged subjects were able to correct intentional disturbances during walking, probably by changing the width of the step, by which the hip joint variability increased in the sagittal plane. The results of these two studies opposed one-another; in the first study [9], increased variability improved walking, while in the second study [8] decreased movement stability using walking poles corrected and improved gait. There has also been a lot of research on skilled performance, with results being in contradiction with the conventional idea that reduced variability tends to boost motor performance. For example, Wagner et al. [10] examined movement variability and skill level in different throwing patterns. They produced different throwing patterns using handball throws and found that the more skilled players would decrease their throwing variability by taking an additional step. This study demonstrated that decreased movement variability at higher skill levels. It should be noted that similar throwing patterns were used in the present study. In contrast to these findings, Wilson et al. [11] looked up at the variability of movements during triple-jumping. They reported that movement variability was greater in both highly skilled jumpers and beginners compared to intermediate jumpers. These results are in contrast with the conventional idea that movement variability decrease with increasing skill level. Wilson et al. [11] reported that the relationship between movement variability and skill level follows an inverse model. The findings reported in the literature vary in this regard in which some studies suggest that reduced movement variability at different levels of the system signify an increase in motor efficiency and skill [12-14], while some researchers have reported otherwise $[15,16]$. In other words, movement variability is not always bad; changes in the components related to motor performance are sometimes advantageous [6]. The question that arises is how can these findings be interpreted? Is variability an advantage in the movement system or does it hinder performance in skilled players? To provide more evidences, a hypothesis can be proposed on the subject of aiming skills in which the relationship between throwing accuracy and joint kinematic changes can explain the degree of variability in a targeting task. If kinematic variations have less entropy with throwing scores, then this variability can be interpreted as an advantage for throws, and if greater entropy prevails, then variability can be considered as a flaw in movement. We used dart throwing and we enrolled skilled darts players with different levels of training to examine the issue at various skill levels.

Hypothesis. Purpose:

- High throwing scores acquire with high variability in elbow angle, velocity and acceleration.

- High throwing scores acquire with low variability in elbow angle, velocity and acceleration.

- High throwing scores acquire with moderate variability in elbow angle, velocity and acceleration.

\section{Material and methods}

\section{Participants}

Seven experienced darts players (three women, four men) with an average training experience of 6.38 years $(\mathrm{M}=6.38, \mathrm{SD}=2.78)$ participated in this study. All players had a minimum of four and maximum of eight years of training experience. Most of the participants were involved in the national darts team, with some being members at the time of the study.

\section{Research Design.}

Materials. Qualisys motion capture system with six cameras was used to record the kinematics of the elbow. The markers, which were silver in colour with diameters of $1.5 \mathrm{~cm}$, were fixed to the shoulder, elbow, and wrist using double-sided tape; these sites of marker placement were selected based on the Helen Hayes marker placement. In addition, a standard dartboard was used according to World Darts Federation (WDF) regulations. Each player used their own standard set of darts in the experiment, such that they had used the same darts for prior training sessions and competitions.

Procedure. Prior to each throw, the markers were fixed 
on the player's elbow and the cameras were calibrated on the throwing zone. First the players were sitting on the chair then markers were installed based on the Helen Hayes marker system. Markers position was detected by touch elbow epicondyle before two markers were installed on the players' right hand external and internal elbow, the cameras were checked again after each set (12 throws) in order to increase the accuracy of the kinematic measuring then the cameras were calibrated again if necessary. Frequency of kinematics measurement was 100 hertz. Trajectory that used to calculate angle was from starting the forearm back movement to when elbow is straight after dart release.

The participants began throwing at their own rhythm whenever they were ready. Before the actual throws, the players took several pilot throws. After declaring their readiness for recording, each participant threw 36 darts over three sets from a 2.37 meters distance. Five-minute rests were devised between each set. Every three throws were regarded as one round, and there was a brief interval between each round. Data related to the middle throw from each round was used in later analysis. Scoring was limited to triangle 20, which was divided into 15 scoring regions, each $1 \mathrm{~cm}$ apart. The highest score of 60 points was achieved by hitting the middle area, while the most distant regions from the centre were worth 25 points; the points decreased by five with each region leaving the centre.

Data processing and analysis. MATLAB software was used for data filtering and processing. First, data was filtered using a band pass $10-500 \mathrm{~Hz}$, fifth-order. Positions of the shoulder and wrist markers were used to calculate

Table 1. Evaluation of the skill level of the participants the normalized values of the angle, angular velocity and angular acceleration of the elbow. In order to investigate the variability in the throw scores, entropy analysis of the throw angle, velocity and acceleration was conducted. SPSS was used for statistical analysis.

\section{Results}

The mean skill level of the participants can be expressed as $53 \%$. In this regard, the percentage of throws that hit the target triangle was considered as the benchmark for skill level. For more information, see table 1.

The entropy of the throwing angle, angular velocity and acceleration was examined. In order to investigate the impact of angular variability on throwing performance, general linear regression models were used to evaluate the participants' scores. At a significance level of 5\%, disregarding the y-intercept, the hypothesis that angular stability has an effect on throwing score was rejected. This means that if the players change their throwing angle in any way, the number of scores obtained will not change. On the other hand, disregarding the y-intercept, which its linear model is later described, it was found that variability in angular changes had a significant effect on the score achieved. The fitted data of the entropy related to the impact of variability in angle, acceleration and velocity on the by scores obtained darts throwers, disregarding the y-intercept, is shown in table 2.

The coefficient of determination for the three models in which the entropies of angle, acceleration and velocity were investigated were $0.53,0.44$ and 0.53 , respectively, which demonstrated the suitability of the models.

\begin{tabular}{lll}
\hline Participant & Number of throws & Skill level (\%) \\
\hline 1 & 12 & $83 \% * * *$ \\
2 & 10 & $50 \% * *$ \\
3 & 12 & $58 \% * *$ \\
4 & 10 & $20 \% *$ \\
5 & 12 & $67 \% * * *$ \\
6 & 12 & $50 \% * *$ \\
7 & 11 & $45 \% * *$ \\
Total / Means & 79 & $53 \% * *$ \\
\hline
\end{tabular}

Table 2. The fitting obtained from the linear model of scores on angle, velocity and acceleration entropy, disregarding the $y$-intercept

\begin{tabular}{lllll}
\hline Parameter & Parameter Estimation & Standard Error & Test statistic & Significance \\
\hline Angle & 5.922 & 0.638 & 9.392 & $0.000^{*}$ \\
Velocity & 5.580 & 0.597 & 9.399 & $0.000^{*}$ \\
Acceleration & 2.106 & 0.270 & 7.799 & $0.000^{*}$ \\
\hline
\end{tabular}

* significant entropy 


\section{Discussion}

The purpose of the present study was to investigate the variability of movement in throwing at high skill levels. The results indicated that for highly skilled individuals who have trained and repeatedly performed a dartthrowing task from a specific distance, optimal throws are achieved by moderate variability. The remarkable point in these findings was that variability was constant across all samples despite the varied range of experience in throwing darts from 2.37 meters distance.

Entropy analysis showed that in the throws of highly experienced individuals, variation led to greater throwing efficiency. The efficiency of the motor system seemed to increase as the variability in throws increased to a certain limit, beyond which the performing components appeared to have trouble maintaining efficiency. The figure 1 clearly demonstrates these findings.

Fitting of the quadratic model demonstrates a U-like performance curve, which supports the idea that throws with moderate variability result in the highest scores which is supported by some previous studies [16]. Button et al. [16] reached at the unexpected conclusion that movement variability does not decrease with increasing skill levels by examining the variability of basketball free throws at different skill levels. They also reported that along with increased skill levels, the, wrist and elbow joint stabilities increased that may refer to compensation of wrist and elbow in one trial [16]. These findings are contrary to the conventional notion that regards movement variability as noise that is indicative of the human motor system's inefficiency $[7,17]$ The findings of the present study are in contrary with those of the recent study of Longo et al [18], who reported that as movement accuracy increases, the kinematic variability of movement decreases [18]. Considering that the dart throwing task revolves around accuracy, the present study was not support that finding since increased variability in elbow angle, angular velocity and acceleration was associated with better throwing performance. Although these changes were only effective to a certain limit, these differing findings may be related to the differences in skill levels (amateur vs. experienced participants). Another possible reason may be the difference in the task examined between the two studies (bimanual reaching vs. dart throwing).

One hypothesis was proposed by Latash et al. to explain the moderate variability as optimal [6]. The need for variation to perform better throws and earn higher scores in this system can be interpreted as the existence of different solutions for the nervous system and the existence of a kind of internal flexibility in the system for creating variables related to performance [19]. These interpretations are in regard to the effect of the internal changes occurring in the elbow on throwing scores, and, given the paired coordination between the operating muscles and joints [20], these changes must be looked at more closely. In this case, it can be said that the internal connections of the elbow markers represent a kind of cooperation between the involved muscles and motor units of the arm and forearm during throwing.

\section{Conclusions}

The results of the present study revealed that numerous repetitions of throwing at a target from a specific distance optimize the movement variability in the kinematics of the elbow joint. These findings show that with increased performance levels, variability within the system becomes a performance improvement factor, but only a moderate amount of variability causes this improvement, beyond which the system is degraded.

\section{Highlights}

- Variability of angle, velocity and acceleration have significance effects on throwing scores.

- variability was constant across all samples despite the varied range of experience

- Optimal throws are achieved by moderate variability in highly skilled individuals who have trained and repeatedly performed a dart-throwing task from a specific distance.

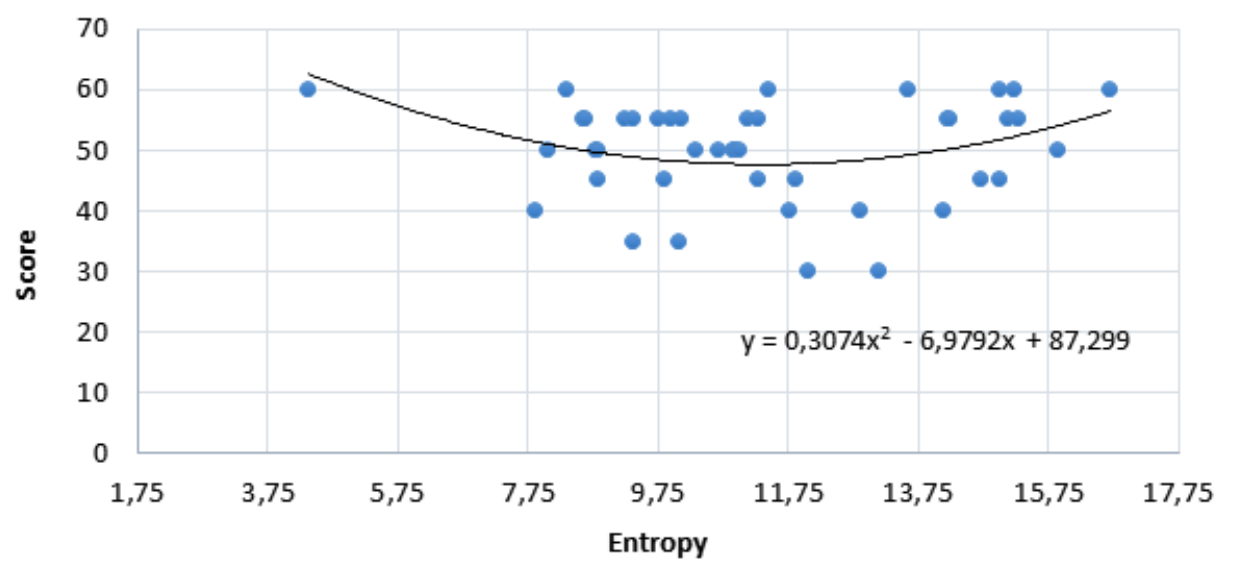

Figure 1. Entropy analysis - The quadratic model (curved line fitted to the effect of variations in throwing darts on scores achieved (blue points) 


\section{Acknowledgment\#}

We must appreciate Mohamad Lavaei for statistical analysis and nice consultant and Mr Hoseini for translate Persian manuscript to English version and later proof.

\section{References}

1. Stergiou N, Harbourne R, Cavanaugh J. Optimal movement variability: a new theoretical perspective for neurologic physical therapy. J Neurol Phys Ther. 2006;30(3):120-9. https://doi.org/10.1097/01.NPT.0000281949.48193.d9

2. Wulf G, Schmidt RA. Feedback-Induced Variability and the Learning of Generalized Motor Programs. J Mot Behav. 1994;26(4):348-61. https://doi.org/10.1080/00222895.1994.9941691

3. Scholz JP, Kang N, Patterson D, Latash ML. Uncontrolled manifold analysis of single trials during multifinger force production by persons with and without Down syndrome. Exp Brain Res. 2003;153(1):45-58. https://doi.org/10.1007/s00221-003-1580-8

4. Schoner G, Kelso J. Dynamic pattern generation in behavioral and neural systems. Science. 1988;239(4847):1513-20. https://doi.org/10.1126/science.3281253

5. Stergiou N, Decker LM. Human movement variability, nonlinear dynamics, and pathology: is there a connection? Hum Mov Sci. 2011;30(5):869-88. https://doi.org/10.1016/j.humov.2011.06.002

6. Latash ML. Movements that are both variable and optimal. J Hum Kinet. 2012;34:5-13. https://doi.org/10.2478/v10078-012-0058-9

7. Latash ML, Anson JG. Synergies in health and disease: relations to adaptive changes in motor coordination. Phys Ther. 2006;86(8):1151-60. https://doi.org/10.1093/ptj/86.8.1151

8. Zoffoli L, Ditroilo M, Federici A, Lucertini F. Local stability and kinematic variability in walking and pole walking at different speeds. Gait \& Posture. 2017;53:1-4. https://doi.org/10.1016/j.gaitpost.2016.12.017

9. Qiao M, Feld JA, Franz JR. Aging effects on leg joint variability during walking with balance perturbations. Gait \& Posture, 2018;62:27-33. https://doi.org/10.1016/j.gaitpost.2018.02.020

10.WagnerH,PfusterschmiedJ, KlousM, vonDuvillardSP, Müller E. Movement variability and skill level of various throwing techniques. Human Movement Science. 2012;31(1):78-90. https://doi.org/10.1016/j.humov.2011.05.005

\section{Funding}

This work was supported by the University of ValiAsr-Rafsanjan (Kerman, Iran)

\section{Conflict of interest}

Author declare no conflict of interest.
11. Wilson C, Simpson SE, Van Emmerik RE, Hamill J. Coordination variability and skill development in expert triple jumpers. Sports Biomechanics. 2008;7(1):2-9. https://doi.org/10.1080/14763140701682983

12.Floría P, Sánchez-Sixto A, Ferber R, Harrison AJ. Effects of running experience on coordination and its variability in runners. Journal of Sports Sciences. 2018;36(3):272-8. https://doi.org/10.1080/02640414.2017.1300314

13.Ko J-H, Han D-W, Newell KM. Skill level changes the coordination and variability of standing posture and movement in a pistol-aiming task. Journal of Sports Sciences. 2018;36(7):809-16. https://doi.org/10.1080/02640414.2017.1343490

14. Serrien B, Goossens M, Baeyens J-P. Proximal-to-Distal Sequencing and Coordination Variability in the Volleyball Spike of Elite Youth Players: Effects of Gender and Growth. Journal of Motor Learning and Development, 2018;6:250-66. https://doi.org/10.1123/jmld.2017-0049

15.Sternad D. It's not (only) the mean that matters: variability, noise and exploration in skill learning. Current Opinion in Behavioral Sciences. 2018;20:183-95. https://doi.org/10.1016/j.cobeha.2018.01.004

16.Button C, MacLeod M, Sanders R, Coleman S. Examining movement variability in the basketball free-throw action at different skill levels. Res Q Exerc Sport. 2003;74(3):257-69. https://doi.org/10.1080/02701367.2003.10609090

17.Horan SA, Evans K, Kavanagh JJ. Movement variability in the golf swing of male and female skilled golfers. Medicine and Science in Sports and Exercise. 2011;43(8):1474-83. https://doi.org/10.1249/MSS.0b013e318210fe03

18.Longo A, Meulenbroek R. Precision-dependent changes in motor variability during sustained bimanual reaching. Motor Control. 2018;22(1):28-44. https://doi.org/10.1123/mc.2016-0013

19.Latash ML, Scholz JF, Danion F, Schoner G. Structure of motor variability in marginally redundant multifinger force production tasks. Exp Brain Res. 2001;141(2):153-65. https://doi.org/10.1007/s002210100861

20.Latash ML, Scholz JP, Schoner G. Toward a new theory of motor synergies. Motor Control. 2007;11(3):276-308. https://doi.org/10.1123/mcj.11.3.276 


\section{Information about the author:}

Mahdi Nabavinik; http://orcid.org/0000-0001-9462-6140; m.nabavinik@umz.ac.ir; University of Mazandaran; Pasdaran Street, 57416-13534, P.O.Box: 416, Babolsar, Iran.

Hamideh Abdolzadeh; (Corresponding Author); http://orcid.org/0000-0001-5919-4557; h.abdolzadeh@vru.ac.ir; Sport science group, University of Vali-Asr-Rafsanjan; Main Administration Building, 22 Bahman Square, Rafsanjan,Iran.

Cite this article as:

Nabavinik M, Hamideh Abdolzadeh. Moderate movement variability is optimal in massive practiced dart throws.

Pedagogy of Physical Culture and Sports, 2020;24(6):297-302. https://doi.org/10.15561/26649837.2020.0604

This is an Open Access article distributed under the terms of the Creative Commons Attribution License, which permits unrestricted use, distribution, and reproduction in any medium, provided the original work is properly cited (http://creativecommons.org/licenses/by/4.0/deed.en).

Received: 28.05.2020

Accepted: 01.07.2020; Published: 30.12.2020 